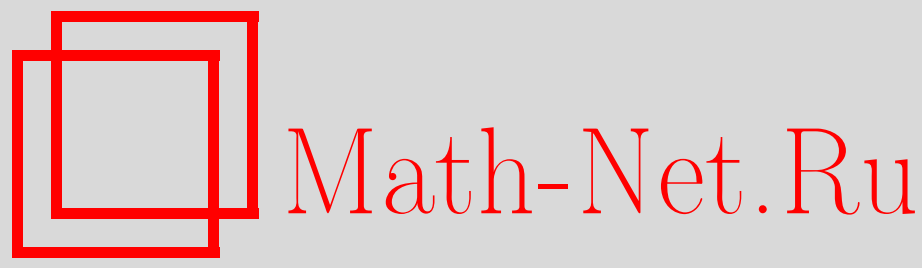

Л. Фехер, Б. Г. Пустай, Гамильтоновы редукции свободных частиц относительно полярных действий компактных групп Ли, ТМФ, 2008, том 155, номер 1, 161-176

DOI: https://doi.org/10.4213/tmf6201

Использование Общероссийского математического портала Math-Net.Ru подразумевает, что вы прочитали и согласны с пользовательским соглашением http://www . mathnet.ru/rus/agreement

Параметры загрузки:

IP : 54.157 .27 .8

26 апреля 2023 г., 18:28:19

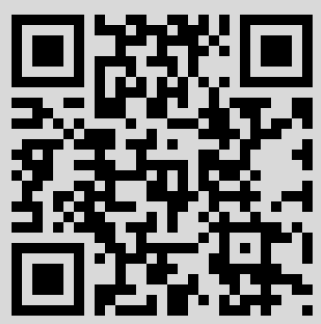




\section{ГАМИЛЬТОНОВЫ РЕДУКЦИИ СВОБОДНЫХ ЧАСТИЦ ОТНОСИТЕЛЬНО ПОЛЯРНЫХ ДЕЙСТВИЙ КОМПАКТНЫХ ГРУПП ЛИ}

Исследуются классические и квантовые гамильтоновы редукции свободных геодезических систем на полных римановых многообразиях. Редуцированные системы описываются в предположении, что соответствующая компактная группа симметрии действует полярным образом в том смысле, что существуют регулярно вложенные замкнутые связные подмногообразия, ортогонально пересекающие все орбиты в конфигурационном пространстве. Гиперполярные действия на группах Ли и на симметрических пространствах приводят к семействам интегрируемых систем типа системы Калоджеро-Сазерленда со спином.

Ключевые слова: гамильтонова редукция, полярное действие, интегрируемые системы.

\section{1. ВВЕДЕНИЕ}

Одним из основных в теории интегрируемых систем является тот факт, что многие интересные модели возникают как гамильтоновы редукции некоторых канонических "свободных" систем, которые очевидным образом интегрируются благодаря большому числу симметрий. Например, известную модель Сазерленда взаимодействующих частиц на прямой, классически определяемую гамильтонианом

$$
\mathcal{H}(q, p)=\frac{1}{2} \sum_{k=1}^{n} p_{k}^{2}+\nu(\nu-1) \sum_{1 \leqslant i<j \leqslant n} \frac{1}{\sin ^{2}\left(q_{i}-q_{j}\right)},
$$

можно рассматривать как редукцию канонической геодезической системы на группе $S U(n)$. Соответствующая симметрия определяется действием группы на себе сопряжениями, и модель с гамильтонианом (1.1) получается (в системе центра

*Department of Theoretical Physics, MTA KFKI RMKI, Budapest; Department of Theoretical Physics, University of Szeged, Szeged, Hungary. E-mail: lfeher@rmki.kfki.hu

${ }^{\dagger}$ Centre de recherches mathématiques, Université de Montréal; Department of Mathematics and Statistics, Concordia University, Montréal, Québec, Canada.

E-mail: pusztai@CRM.UMontreal.CA 
масс) после фиксирования нётеровских зарядов этой симметрии специальным образом. Гамильтонову редукцию можно выполнить как на классическом [1], так и на квантово-механическом [2] уровне. С помощью теории представлений в последнем случае получается модель с целочисленными значениями константы взаимодействия $\nu$. Вывод модели Сазерленда с гамильтонианом (1.1) с помощью гамильтоновой редукции был обобщен на случаи, когда получаются другие интегрируемые модели типа модели Калоджеро-Мозера-Сазерленда (в том числе со спином) [3]-[13].

Наиболее прямые обобщения [6], [7] получаются при замене группы $S U(n)$ на произвольную связную компактную простую группу Ли и фиксации нётеровских зарядов произвольным образом. Тогда редукции, основанные на симметрии относительно сопряжений, приводят к моделям типа модели Сазерленда со спином с потенциалом взаимодействия, определяемым соответствующей системой корней. На классическом уровне фазовое пространство этих моделей содержит дополнительные ("спиновые") степени свободы в дополнение к кокасательному расслоению редуцированного конфигурационного пространства, представляющего координаты частиц. На квантовом уровне гамильтониан действует на многокомпонентные волновые функции, а не на скалярные волновые функции в моделях типа бесспиновой модели Сазерленда. Эти волновые функции соответствуют векторнозначным сферическим функциям на группе симметрии, изучению которых уделяется много внимания в гармоническом анализе.

Другие обобщения исходят из геодезических систем на симметрических пространствах. Первые результаты в этом направлении исследований принадлежат Ольшанецкому и Переломову [3], [4] (по поводу последних достижений см., например, [11]-[13] и приведенную там библиографию).

В приведенных примерах с исходными данными всегда ассоциируется связное полное риманово многообразие, снабженное изометрическим действием компактной группы симметрии, которая допускает плоские сечения. Сечение (в смысле Пале и Тернга [14]) для изометрического действия группы $G$ есть регулярно вложенное замкнутое связное подмногообразие, которое пересекает каждую $G$-орбиту по крайней мере один раз и притом ортогонально в каждой точке пересечения. Действие, допускающее сечения, называется полярным, а полярные действия с плоскими сечениями называются гиперполярными. Например, действие сопряжениями связной компактной группы Ли на себе является гиперполярным, а сечениями являются максимальные торы. Полярные действия, в особенности на симметрических пространствах, много изучались в литературе [15]-[18].

Цель данной работы - представить некоторые общие результаты о гамильтоновой симметрийной редукции относительно полярных действий компактных групп Ли. Мотивировка обусловлена нашим интересом к интегрируемым системам, для которых примеры таких редукций уже оказались полезными, как это было отмечено выше. С одной стороны, мы осознали, что и классическую, и квантовую гамильтонову редукцию легко рассматривать единым способом в рамках формализма полярных действий. С другой стороны, оказывается, что редуцированные систе- 
мы, возникающие из гиперполярных действий на группах Ли и на симметрических пространствах, приводят, вообще говоря, к интегрируемым моделям типа модели Калоджеро-Сазерленда со спином. Данная статья может служить шагом на пути к систематическому исследованию этих моделей.

План статьи и основные результаты таковы. В разделе 2 мы сначала напоминаем некоторые предварительные сведения, а затем обращаемся к классической гамильтоновой редукции геодезического движения, уделяя основное внимание плотному открытому подмногообразию регулярных элементов в конфигурационном пространстве. Основным результатом раздела 3 является теорема 1, которая характеризует редуцированные системы, возникающие при произвольном значении отображения моментов. Этот результат можно получить также с помощью более общих результатов Хохгернера [10], [19] о редуцированных кокасательных расслоениях. Мы даем его простое, независимое доказательство непосредственно в рамках формализма полярных действий. Раздел 4 посвящен квантовой гамильтоновой редукции. По сути дела она сводится к ограничению оператора Лапласа-Бельтрами риманова многообразия на некоторые эквивариантные волновые функции. Здесь нашим основным результатом является теорема 2, в которой приводятся как явный вид редуцированного оператора Лапласа-Бельтрами, так и его самосопряженность в подходящей области. В разделе 5 рассмотрены некоторые примеры гиперполярных действий, которые приводят к моделям типа модели Калоджеро-Сазерленда со спином; это будет более подробно рассмотрено в другой публикации.

Хотя некоторые из приведенных нами результатов или их специальные случаи уже известны, они, по-видимому, не рассматривались с единой точки зрения полярных действий. Мы сочли полезным систематизировать и расширить существующие результаты с этих позиций. Подчеркнем, что мы представляем классическую и квантовую гамильтоновы редукции как два разнообразных подхода к одной задаче, показывая как сходства, так и различия между соответствующими классическими и квантовыми редуцированными системами.

\section{2. ОСНОВНЫЕ ПРЕДПОЛОЖЕНИЯ И ОПРЕДЕЛЕНИЯ}

Рассмотрим гладкое связное полное риманово многообразие $Y$ с метрикой $\eta$ и компактную группу Ли $G$, которая действует как группа симметрии многообразия $(Y, \eta)$. Особо удобна ситуация, когда групповое действие допускает сечения в том виде, в каком они определены и исследованы в работе [14]. По определению сечение $\Sigma$ является связным замкнутым регулярно вложенным гладким подмногообразием в $Y$, которое пересекает каждую $G$-орбиту и притом ортогонально в любой точке пересечения. Как уже отмечалось, групповые действия, допускающие сечения, называются полярными, а если сечения являются плоскими в индуцированной метрике, то они называются гиперполярными. (Некоторые авторы различными способами ослабляют определение, например от сечений иногда требуется, чтобы они были только иммерсированными подмногообразиями.) 
Пусть $\check{Y}$ обозначает открытое плотное $G$-инвариантное подмногообразие в $Y$, состоящее из $G$-регулярных точек типа главной орбиты ${ }^{1)}$, т.е. тех точек $y \in Y$, подгруппы изотропии которых $G_{y}$ являются наименьшими возможными для данного $G$-действия $G \ni g \mapsto \phi_{g} \in \operatorname{Diff}(Y)$. Поскольку подгруппы изотропии всех элементов из $\check{Y}$ сопряжены в $G$, пространство орбит $\check{Y} / G$ является гладким многообразием, несущим естественную индуцированную риманову метрику, которую мы обозначим как $\eta_{\text {red. }}$ Пусть $\check{\Sigma}$ обозначает связную компоненту многообразия, $\widehat{\Sigma}:=\check{Y} \cap \Sigma$, для сечения $\Sigma$. Известно, что $\check{Y} / G$ является связным, а ограничение естественной проекции $\pi: \check{Y} \rightarrow \check{Y} / G$ на $\check{\Sigma}$ является изометрическим диффеоморфизмом, причем метрика на $\check{\Sigma}$ индуцируется тем, что $\check{\Sigma}-$ подмногообразие в $Y$. Подгруппы изотропии всех элементов в $\widehat{\Sigma}$ одинаковы, и для фиксированного сечения мы определим $K:=G_{y}$ при $y \in \widehat{\Sigma}$.

Стоит заметить, что если сечение существует, то имеется единственное сечение, проходящее через каждую точку $y \in \check{Y}$, которое является образом ортогонального дополнения пространства $T_{y}(G . y)$ в $T_{y} Y$ при геодезическом экспоненциальном отображении. Более того, имеется обобщенная группа Вейля $W(\Sigma)=N(\Sigma) / K$, где $N(\Sigma)$ - подгруппа в $G$, состоящая из тех элементов, которые отображают $\Sigma$ в $\Sigma$, включая $K$ в качестве нормальной подгруппы конечного индекса. Группа $W(\Sigma)$ переставляет связные компоненты многообразия $\widehat{\Sigma}$.

Очень простой пример дается стандартным действием группы $S O(2)$ на плоскости, при котором сечениями являются прямые линии, проходящие через начало координат. В этом случае $\Sigma \backslash \widehat{\Sigma}$ есть просто начало координат и $W \simeq \mathbb{Z}_{2}$. Термин "полярное действие" призван напоминать о наличии "полярных координат." Много интересных примеров можно найти, например, в [17], [18].

Для наших целей мы выберем $G$-инвариантное положительно-определенное скалярное произведение $\mathcal{B}$ на $\mathcal{G}:=\operatorname{Lie}(G)$ и с помощью $\mathcal{B}$ будем, как правило, отождествлять $\mathcal{G}$ с ее дуальным пространством $\mathcal{G}^{*}$. Имеем алгебры Ли $\mathcal{G}_{y}:=\operatorname{Lie}\left(G_{y}\right)$, в частности $\mathcal{K}:=\operatorname{Lie}(K)$, и соответствующие ортогональные разложения

$$
\mathcal{G}=\mathcal{G}_{y} \oplus \mathcal{G}_{y}^{\perp} \quad \forall y \in Y
$$

индуцированные посредством $\mathcal{B}$. Они порождают отождествления $\left(\mathcal{G}_{y}\right)^{*} \simeq \mathcal{G}_{y}$, $\left(\mathcal{G}_{y}^{\perp}\right)^{*} \simeq \mathcal{G}_{y}^{\perp}$. Разложим касательное пространство $T_{y} Y$ на вертикальное и горизонтальное подпространства:

$$
T_{y} Y=V_{y} \oplus H_{y}, \quad V_{y}:=T_{y}(G . y), \quad H_{y}:=V_{y}^{\perp},
$$

где ортогональность определяется с помощью $\eta_{y}$. Используя $\eta_{y}$, отождествим $T_{y} Y$ с его дуальным пространством $T_{y}^{*} Y$, в силу чего имеем $V_{y}^{*} \simeq V_{y}$ и $H_{y}^{*} \simeq H_{y}$. В регулярных точках имеем также

$$
H_{y}=T_{y} \widehat{\Sigma}=T_{y} \Sigma \quad \forall y \in \widehat{\Sigma} .
$$

\footnotetext{
1) Мы называем точки из $\check{Y}$ просто регулярными. По поводу общих ссылок о действиях групп см., например, [20]. Отметим также, что в понятие многообразия мы включаем вторую аксиому счетности.
} 
Для любой $\zeta \in \mathcal{G}$ через $\zeta_{Y}$ обозначим ассоциированное векторное поле на $Y$. Введем отображение

$$
\mathcal{L}_{y}: \mathcal{G} \rightarrow T_{y} Y, \quad \mathcal{L}_{y}: \zeta \mapsto \zeta_{Y}(y)
$$

Ограниченное отображение

$$
\overline{\mathcal{L}}_{y}: \mathcal{G}_{y}^{\perp} \rightarrow V_{y}
$$

является линейным изоморфизмом. Введем также обратное к нему отображение

$$
\bar{A}_{y}:=\left(\overline{\mathcal{L}}_{y}\right)^{-1}: V_{y} \rightarrow \mathcal{G}_{y}^{\perp}
$$

и механическую связность $A_{y}: T_{y} Y \rightarrow \mathcal{G}$, которая продолжает $\bar{A}_{y}$ нулем на $H_{y}$. Далее определим оператор инерции $\mathcal{I}_{y} \in \operatorname{End}\left(\mathcal{G}_{y}^{\perp}\right)$ условием, что

$$
\eta_{y}\left(\mathcal{L}_{y} \xi, \mathcal{L}_{y} \zeta\right)=\mathcal{B}\left(\mathcal{I}_{y} \xi, \zeta\right) \quad \forall \xi, \zeta \in \mathcal{G}_{y}^{\perp}
$$

Оператор инерции является симметричным и положительно-определенным по отношению к ограничению скалярного произведения $\mathcal{B}$ на $\mathcal{G}{ }_{y}^{\perp}$. Он обладает свойством эквивариантности

$$
\mathcal{I}_{\phi_{g}(y)} \circ \mathcal{M}_{g}=\mathcal{M}_{g} \circ \mathcal{I}_{y}
$$

где $\mathcal{M}_{g}: \mathcal{G}_{y}^{\perp} \rightarrow \mathcal{G}_{\phi_{g}(y)}^{\perp}$ задано как $\mathcal{M}_{g}(\xi)=\operatorname{Ad}_{g}(\xi)$.

Подъем действия группы $G$ на кокасательное расслоение $T^{*} Y$ является гамильтоновым по отношению к канонической симплектической структуре, а ассоциированное отображение моментов

$$
\psi: T^{*} Y \rightarrow \mathcal{G}^{*}
$$

задается формулой

$$
\psi\left(\alpha_{y}\right)=\mathcal{L}_{y}^{*}\left(\alpha_{y}\right) \quad \forall \alpha_{y} \in T_{y}^{*} Y
$$

откуда следует, что $\psi\left(\alpha_{y}\right) \in \mathcal{G}_{y}^{0} \simeq \mathcal{G}_{y}^{\perp}$, где $\mathcal{G}_{y}^{0} \subset \mathcal{G}^{*}-$ аннулятор $\mathcal{G}_{y}$.

\section{3. КЛАССИЧЕСКАЯ ГАМИЛЬТОНОВА РЕДУКЦИЯ}

Геодезическое движение на римановом многообразии $(Y, \eta)$ можно моделировать как гамильтонову систему на $T^{*} Y$. Важной задачей является описание симметрийных редукций такой системы, основанных на изометрических собственных групповых действиях. Этот вопрос на данный момент все еще открыт ввиду сложности, связанной с особенностями, присущими $Y / G$. Ограничиваясь на тип главных орбит, всегда (независимо от наличия или отсутствия сечений) получаем гладкое редуцированное конфигурационное пространство $\check{Y}_{\text {red }}:=\check{Y} / G$. Поэтому симметрийные редукции расслоения $T^{*} \check{Y}$ характеризуются намного проще. Хохгернер [10], [19] дал общий анализ редукций кокасательного расслоения в предположении единого типа изотропии для действия на конфигурационном пространстве. Теорема 1 следует как специальный случай из его результата, когда $G$-действие допускает сечения. Для удобства, поскольку общий случай достаточно непрост, мы дадим прямое доказательство в нашем более частном случае. 
Оказывается удобным использовать “сдвиговый трюк” симплектической редукции [21] для характеризации (сингулярных) редукций Марсдена-Вайнстайна геодезической системы на $T^{*} \check{Y}$. Другими словами, возьмем коприсоединенную орбиту $\mathcal{O}$ группы $G$, для которой $-\mathcal{O} \subset \psi\left(T^{*} \check{Y}\right)$, и будем исходить из расширенной гамильтоновой системы

$$
\left(\check{P}^{\text {ext }}, \Omega^{\text {ext }}, \mathcal{H}^{\text {ext }}\right)
$$

определяемой следующим образом. Расширенное фазовое пространство

$$
\check{P}^{\text {ext }}:=T^{*} \check{Y} \times \mathcal{O}=\left\{\left(\alpha_{y}, \xi\right) \mid \alpha_{y} \in T_{y}^{*} \check{Y}, y \in \check{Y}, \xi \in \mathcal{O}\right\}
$$

снабжено симплектической структурой произведения

$$
\Omega^{\operatorname{ext}}\left(\alpha_{y}, \xi\right)=\left(d \theta_{\check{Y}}\right)\left(\alpha_{y}\right)+\omega(\xi)
$$

где $\theta_{\check{Y}}$ - каноническая 1-форма расслоения $T^{*} \check{Y}$, а $\omega-$ симплектическая форма орбиты $\mathcal{O}$. Расширенный гамильтониан является просто кинетической энергией

$$
\mathcal{H}^{\mathrm{ext}}\left(\alpha_{y}, \xi\right):=\frac{1}{2} \eta_{y}^{*}\left(\alpha_{y}, \alpha_{y}\right)
$$

где $\eta_{y}^{*}$ - метрика на $T_{y}^{*} \check{Y}$, соответствующая метрике $\eta_{y}$ на $T_{y} \check{Y}$. Для всех $g \in G$ обозначим через $\phi_{g}^{\text {ext }}$ естественное диагональное действие группы $G$ на $P^{\text {ext }}$. Это действие осуществляется комбинированием кокасательного подъема исходного действия $\phi_{g}$ с коприсоединенным действием на орбите $\mathcal{O}$, а потому (если $\psi$ определено в (2.10)) ассоциированное отображение моментов реализуется как

$$
\Psi: \check{P}^{\mathrm{ext}} \rightarrow \mathcal{G}^{*}, \quad \Psi\left(\alpha_{y}, \xi\right)=\psi\left(\alpha_{y}\right)+\xi
$$

Наша задача состоит в описании редуцированной гамильтоновой системы при $\Psi=0$, для чего мы используем обозначение

$$
\left(\check{P}_{\text {red }}, \Omega_{\text {red }}, \mathcal{H}_{\text {red }}\right) \quad \text { при } \quad \check{P}_{\text {red }}=\check{P}^{\text {ext }} / /{ }_{0} G:=\check{P}_{\Psi=0}^{\text {ext }} / G \text {. }
$$

В связи с обозначениями из раздела 2 заметим, что главная группа изотропии $K \subset G$ естественным образом действует на орбите $\mathcal{O}$, и отображение моментов для этого действия является проекцией $\left.\mathcal{G}^{*} \ni \xi \mapsto \xi\right|_{\mathcal{K}} \in \mathcal{K}^{*}$. Введем тогда (сингулярную) редуцированную коприсоединенную орбиту

$$
\left(\mathcal{O}_{\text {red }}, \omega_{\text {red }}\right) \quad \text { при } \quad \mathcal{O}_{\text {red }}=\mathcal{O} / /{ }_{0} K \simeq\left(\mathcal{O} \cap \mathcal{K}^{\perp}\right) / K,
$$

где в последнем равенстве мы отождествили $\mathcal{G}^{*}$ с $\mathcal{G}$ с помощью $\mathcal{B}$. Редуцированная орбита является, вообще говоря, стратифицированным симплектическим пространством, т.е. объединением гладких симплектических многообразий (см. [21]). Реду-

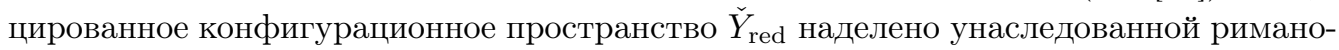
вой метрикой $\eta_{\mathrm{red}}$; обозначим через $\eta_{\mathrm{red}}^{*}$ соответствующее скалярное произведение на слоях расслоения $T^{*} \check{Y}_{\text {red }}$. Наконец, пусть $\theta_{\check{Y}_{\text {red }}}-$ естественная 1 -форма на $T^{*} \check{Y}_{\text {red }}$. 
Теорема 1 [10]. Предположим, что $G$-действие на $(Y, \eta)$ допускает сечения. Фиксируем связную компоненту $\check{\Sigma}$ регулярных элементов сечения $\Sigma$. Тогда для любой орбиты $-\mathcal{O} \subset \psi\left(T^{*} \check{Y}\right)$ редуцированное фазовое пространство (3.6) можно представить как

$$
\check{P}_{\text {red }}=T^{*} \check{Y}_{\text {red }} \times \mathcal{O}_{\text {red }}=\left\{\left(p_{q},[\xi]\right) \mid p_{q} \in T_{q}^{*} \check{Y}_{\text {red }}, q \in \check{Y}_{\text {red }},[\xi] \in \mathcal{O}_{\text {red }}\right\} .
$$

При этом оно снабжается (стратифицированной) симплектической структурой произведения

$$
\Omega_{\text {red }}\left(p_{q},[\xi]\right)=\left(d \theta_{\check{Y}_{\text {red }}}\right)\left(p_{q}\right)+\omega_{\text {red }}([\xi]) .
$$

Редуцированный гамильтониан, возникающий из кинетической энергии геодезического движения на $\check{Y}$, имеет вид

$$
\mathcal{H}_{\text {red }}\left(p_{q},[\xi]\right)=\frac{1}{2} \eta_{\text {red }}^{*}\left(p_{q}, p_{q}\right)+\frac{1}{2} \mathcal{B}\left(\mathcal{I}_{y(q)}^{-1} \xi, \xi\right),
$$

где $\eta_{\text {red }}-$ индуцированная метрика на $\check{Y}_{\text {red }}, y(q) \in \check{\Sigma}-$ проектор на $q \in \check{Y}_{\text {red }},[\xi]=$ $K . \xi \subset \mathcal{O} \cap \mathcal{K}^{\perp}, a \mathcal{I}_{y(q)} \in G L\left(\mathcal{K}^{\perp}\right)-K$-эквивариантный оператор инерции (2.7).

ДокАЗАТЕЛЬСТво. Ключевым моментом при описании редуцированной системы (3.6) является введение подмногообразия $S \subset \check{P}_{\Psi=0}^{\text {ext }}$,

$$
S=\left\{\left(\alpha_{y}, \xi\right) \in \check{P}_{\Psi=0}^{\mathrm{ext}} \mid y \in \check{\Sigma}\right\} .
$$

Bce $G$-орбиты в $\check{P}_{\Psi=0}^{\text {ext }}$ пересекают $S$ и остаточные калибровочные преобразования, соответствующие этому подмногообразию, порождаются группой $K$. Действительно, элемент группы $G$ может отобразить некоторый элемент из $S$ в $S$, только если он принадлежит $K$, а элементы $K$ отображают каждый элемент из $S$ в $S$. В результате редуцированное фазовое пространство обладает следующим свойством:

$$
\check{P}_{\text {red }}=\check{P}_{\Psi=0}^{\text {ext }} / G=S / K \text {. }
$$

Редуцированная симплектическая структура и редуцированный гамильтониан получаются простым разрешением связи на отображение моментов $\psi\left(\alpha_{y}\right)+\xi=0$ на $S$. Для этого рассмотрим ортогональные разложения

$$
\alpha_{y}=\alpha_{y}^{H}+\alpha_{y}^{V}, \quad \xi=\xi_{\mathcal{K}}+\xi_{\mathcal{K}^{\perp}},
$$

соответствующие $(2.2)$ и $\mathcal{G}=\mathcal{K} \oplus \mathcal{K}^{\perp}$ из (2.1). Легко видеть, что связь на отображение моментов полностью разрешается на $S$ с помощью соотношений

$$
\xi_{\mathcal{K}}=0, \quad y \in \check{\Sigma}, \quad \alpha_{y}^{H} \in H_{y}^{*} \text { произвольно, }
$$

и формулы

$$
\alpha_{y}^{V}=-\bar{A}_{y}^{*}\left(\xi_{\mathcal{K}^{\perp}}\right)
$$

где мы отождествили $\mathcal{G}$ с $\mathcal{G}^{*}$ с помощью $\mathcal{B}$ и использовали механическую связность $\bar{A}_{y}: V_{y} \rightarrow \mathcal{K}^{\perp}(2.6)$. Заметим, что элемент $\alpha_{y}^{H} \in H_{y}^{*} \simeq T_{y}^{*} \check{\Sigma}(2.3)$ можно естественным образом рассматривать как принадлежий кокасательному расслоению $T^{*} \check{\Sigma}$, и параметризация $S$ с помощью переменных $\left(\alpha_{y}^{H}, \xi_{\mathcal{K}^{\perp}}\right)$ приводит к отождествлению

$$
S \simeq T^{*} \check{\Sigma} \times\left(\mathcal{O} \cap \mathcal{K}^{\perp}\right)=\left\{\left(\alpha_{y}^{H}, \xi_{\mathcal{K}^{\perp}}\right) \mid \alpha_{y}^{H} \in T_{y}^{*} \check{\Sigma}, y \in \check{\Sigma}, \xi_{\mathcal{K}^{\perp}} \in \mathcal{O} \cap \mathcal{K}^{\perp}\right\} .
$$


Это отождествление $K$-эквивариантно, поскольку $\bar{A}_{y}^{*}-K$-эквивариантное отображение, а $\alpha_{y}^{H}-K$-инвариант, так как $K$ является группой изотропии всех $y \in \check{\Sigma}$ (и "срезовое представление" всегда тривиально в регулярных точках). Теперь первое важное обстоятельство состоит в том, что в терминах отождествления (3.16) обратный образ $\left.\Omega^{\mathrm{ext}}\right|_{S}$ структуры $\Omega^{\mathrm{ext}}$ на $S$ принимает вид

$$
\left.\Omega^{\mathrm{ext}}\right|_{S}\left(\alpha_{y}^{H}, \xi_{\mathcal{K}^{\perp}}\right)=\left(d \theta_{\check{\Sigma}}\right)\left(\alpha_{y}^{H}\right)+\left.\omega\right|_{\mathcal{O} \cap \mathcal{K}^{\perp}}\left(\xi_{\mathcal{K}^{\perp}}\right)
$$

где $\theta_{\check{\Sigma}}$ - естественная 1 -форма на $T^{*} \check{\Sigma}$. Поскольку $T^{*} \check{\Sigma}$ является моделью $T^{*} \check{Y}_{\text {red }}$, получаем утверждение теоремы относительно редуцированной симплектической структуры. Второе важное обстоятельство состоит в том, что ограничение гамильтониана (3.4) дает в терминах переменных $\left(\alpha_{y}^{H}, \xi_{\mathcal{K}^{\perp}}\right)$ следующую функцию:

$$
\left.\mathcal{H}^{\text {ext }}\right|_{S}\left(\alpha_{y}^{H}, \xi_{\mathcal{K}^{\perp}}\right)=\frac{1}{2} \eta_{y}^{*}\left(\alpha_{y}^{H}, \alpha_{y}^{H}\right)+\frac{1}{2} \eta_{y}^{*}\left(\bar{A}_{y}^{*}\left(\xi_{\mathcal{K}^{\perp}}\right), \bar{A}_{y}^{*}\left(\xi_{\mathcal{K}^{\perp}}\right)\right)
$$

Первый член представляет собой кинетическую энергию частицы на пространстве $\left(\check{Y}_{\text {red }}, \eta_{\text {red }}\right)$, моделируемом подмногообразием $\check{\Sigma}$ в $\check{Y}$. Из определений в разделе 2 непосредственно следует, что второй член можно переписать в виде

$$
\eta_{y}^{*}\left(\bar{A}_{y}^{*}\left(\xi_{\mathcal{K}^{\perp}}\right), \bar{A}_{y}^{*}\left(\xi_{\mathcal{K}^{\perp}}\right)\right)=\mathcal{B}\left(\mathcal{I}_{y}^{-1} \xi_{\mathcal{K}^{\perp}}, \xi_{\mathcal{K}^{\perp}}\right)
$$

что дает корректно определенную функцию на $\check{P}_{\text {red }}$, поскольку (в силу (2.8)) оператор инерции $\mathcal{I}_{y} K$-эквивариантен при $y \in \check{\Sigma}$.

ЗАмечАниЕ 1 . Отождествляя $\check{Y}_{\text {red }}$ с $\check{\Sigma}$, как в доказательстве теоремы 1 , можно также моделировать редуцированное фазовое пространство как $T^{*} \check{\Sigma} \times \mathcal{O}_{\text {red }}$. Более того, можно рассматривать его как фактор-пространство $T^{*} \widehat{\Sigma} \times \mathcal{O}_{\text {red }}$ по естественному диагональному действию конечной группы $W(\Sigma)$. Здесь $W(\Sigma)=N(\Sigma) / K$ действует на $\mathcal{O} / /{ }_{0} K$, поскольку $K$ является нормальной подгруппой в $N(\Sigma)$. Гамильтониан порождает $W(\Sigma)$-инвариантную функцию на $T^{*} \widehat{\Sigma} \times \mathcal{O}_{\text {red }}$. Поэтому все объекты, относящиеся к редуцированной системе, можно интерпретировать как $W(\Sigma)$-инварианты системы, ассоциированной с (регулярной) частью среза $\Sigma$. Это хорошо известно из примеров моделей типа модели Калоджеро (со спином), изучавшихся, например, в [7], [10]-[12].

ЗАмЕчАниЕ 2. Первый член в редуцированном гамильтониане (3.10) является просто кинетической энергией на $\left(\check{Y}_{\text {red }}, \eta_{\text {red }}\right)$, а второй член можно рассматривать как потенциальную энергию, зависящую также от "спиновых" переменных, принадлежащих $\mathcal{O}_{\text {red. }}$ Согласно (3.19) второй член можно также выразить в терминах связности, двойственной к механической, и в определенных случаях функция $q \mapsto \bar{A}_{y(q)}^{*}$ приводит к так называемой динамической $r$-матрице [10], [11]. В некоторых исключительных случаях [1], [12], [13] редуцированная орбита $\mathcal{O}_{\text {red }}$ состоит из одной точки, что означает, что в редуцированной динамике не появляется никаких спиновых степеней свободы. 


\section{4. КВАНТОВАЯ ГАМИЛЬТОНОВА РЕДУКЦИЯ}

Напомним сначала стандартное квантование гамильтоновой системы $\left(P^{\mathrm{ext}}, \Omega^{\mathrm{ext}}, \mathcal{H}^{\mathrm{ext}}\right)$, определяемой аналогично (3.1) при $P^{\mathrm{ext}}=T^{*} Y \times \mathcal{O}$. Налагая затем аналог связей первого рода $\Psi=0$ на гильбертовом пространстве этой системы, мы получим редуцированную квантовую систему. Наконец, мы обсудим соотношение между результатом процедуры “сначала квантуем, потом редуцируем" и структурой редуцированной классической системы, приведенной в теореме 1. Наши рассуждения близки к работе [22], но в контексте полярных действий о редуцированных системах можно сказать больше.

Рассмотрим унитарное представление

$$
\rho: G \rightarrow U(V)
$$

на конечномерном комплексном гильбертовом пространстве $V$ со скалярным произведением $(\cdot, \cdot)_{V}$ и ассоциированное представление алгебры Ли

$$
\rho^{\prime}: \mathcal{G} \rightarrow u(V),
$$

где $u(V)$ - алгебра Ли антиэрмитовых операторов на $V$. Представление $\rho$ можно рассматривать как квантово-механический аналог коприсоединенной орбиты $\mathcal{O}$, которая возникает на классическом уровне. Стандартным квантово-механическим ана-

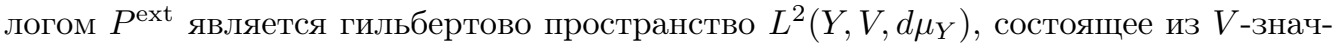
ных квадратично-интегрируемых функций на $Y$ со скалярным произведением

$$
\left(\mathcal{F}_{1}, \mathcal{F}_{2}\right)=\int_{Y}\left(\mathcal{F}_{1}, \mathcal{F}_{2}\right)_{V} d \mu_{Y}
$$

где $d \mu_{Y}$ - мера, ассоциированная с римановой метрикой $\eta$ на $Y$. Пусть $\Delta_{Y}^{0}-$ оператор Лапласа-Бельтрами $\Delta_{Y}$ для $(Y, \eta)$ на области $C_{c}^{\infty}(Y, V) \subset L^{2}\left(Y, V, d \mu_{Y}\right)$, содержащей гладкие $V$-значные функции с компактным носителем. Хорошо известно ${ }^{2)}$, что $\Delta_{Y}^{0}$ существенно самосопряжен, а его замыкание умноженное на $-1 / 2$, дает оператор Гамильтона, соответствующий классическому гамильтониану $\mathcal{H}^{\text {ext }}$.

Нам потребуются эквивариантные функции $\mathcal{F} \in C^{\infty}(Y, V)^{G}$, которые по определению удовлетворяют условию

$$
\mathcal{F} \circ \phi_{g}=\rho(g) \circ \mathcal{F} \quad \forall g \in G .
$$

Аналогичным образом можно определить пространство функций $C^{\infty}\left(\Sigma, V^{K}\right)^{W}$, где $V^{K}$ - подпространство $K$-инвариантных векторов в $V$, на котором $W$ действует в силу того, что $W=N(\Sigma) / K$. Здесь и далее мы полагаем, что $\operatorname{dim}\left(V^{K}\right)>0$. Легко видеть, что ограничение заданных на $Y$ функций на $\Sigma$ порождает инъективное отображение ${ }^{3)}$

$$
C^{\infty}(Y, V)^{G} \longrightarrow C^{\infty}\left(\Sigma, V^{K}\right)^{W}
$$

2) См., например, [23], параграф II.3.7 и приведенную там библиографию. Мы не включаем скаляр Риччи в квантовый гамильтониан, но его включение не привело бы ни к каким осложнениям в нашей аргументации.

3) Известно, что это отображение сюръективно [14], если $\operatorname{dim}(V)=1$. Интересно было бы обобщить этот результат. Другой важный вопрос состоит в нахождении всех случаев, когда $\operatorname{dim}(V)>\operatorname{dim}\left(V^{K}\right)=1$, как в примерах в [2], [9]. 
Отметим еще, что всякая функция $\mathcal{F} \in C^{\infty}(Y, V)^{G}$ единственным образом определена своим ограничением на связную открытую компоненту $\check{\Sigma} \subset \Sigma$. Введем далее линейное пространство

$$
\operatorname{Fun}\left(\check{\Sigma}, V^{K}\right):=\left\{f \in C^{\infty}\left(\check{\Sigma}, V^{K}\right)\left|\exists \mathcal{F} \in C_{c}^{\infty}(Y, V)^{G}, f=\mathcal{F}\right|_{\check{\Sigma}}\right\}
$$

В силу своего изоморфизма с $C_{c}^{\infty}(Y, V)^{G} \subset L^{2}\left(Y, V, d \mu_{Y}\right)$ пространство $\operatorname{Fun}\left(\check{\Sigma}, V^{K}\right)$ становится предгильбертовым. Пусть $\overline{\operatorname{Fun}}\left(\check{\Sigma}, V^{K}\right)$ обозначает его замыкание. Нетрудно проверить, что имеет место естественный изометрический изоморфизм

$$
\overline{\operatorname{Fun}}\left(\check{\Sigma}, V^{K}\right) \simeq L^{2}\left(Y, V, d \mu_{Y}\right)^{G} .
$$

Стоит также заметить, что $\operatorname{Fun}\left(\check{\Sigma}, V^{K}\right)$ содержит $C_{c}^{\infty}\left(\check{\Sigma}, V^{K}\right)$.

В силу (4.7) естественный квантово-механический аналог классической гамильтоновой редукции получается при рассмотрении редуцированного гильбертова пространства в виде $\overline{\operatorname{Fun}}\left(\check{\Sigma}, V^{K}\right)$. Редуцированный оператор Гамильтона получается из $\Delta_{Y}^{0}$ с учетом следующего простого наблюдения. Существует единственный линейный оператор

$$
\Delta_{\mathrm{eff}}: \operatorname{Fun}\left(\check{\Sigma}, V^{K}\right) \rightarrow \operatorname{Fun}\left(\check{\Sigma}, V^{K}\right)
$$

определяемый свойством

$$
\Delta_{\text {eff }} f=\left.\left(\Delta_{Y} \mathcal{F}\right)\right|_{\Sigma} \quad \text { при } \quad f=\left.\mathcal{F}\right|_{\Sigma}, \quad \mathcal{F} \in C_{c}^{\infty}(Y, V)^{G} .
$$

Другими словами, эффективный оператор Лапласа-Бельтрами $\Delta_{\text {eff }}$ является ограничением $\Delta_{Y}$ на $C_{c}^{\infty}(Y, V)^{G}$, которое определено корректно, потому что метрика $\eta$ $G$-инвариантна. Далее мы представим явные формулы для $\Delta_{\text {eff }}$.

4.1. Эффективный оператор Лапласа-Бельтрами. Удобное локальное разложение оператора Лапласа-Бельтрами на радиальную и орбитальную (угловую) части всегда применимо, если имеется локальное ортогональное сечение $G$-орбит на римановом $G$-многообразии [24]. После ограничения на $G$-эквивариантные функции орбитальную часть можно вычислить явно, и в нашем случае можно применить это разложение для $\check{Y}$, поскольку мы имеем дело с полярным действием. Прежде чем описывать результат, нам потребуется ввести новые обозначения.

Рассматривая $\check{\Sigma}$ как гладкую часть редуцированного конфигурационного пространства, мы обозначим через $q$ элементы из $\check{\Sigma}$ и рассмотрим $G$-орбиту $G . q$ через любой элемент $q \in \check{\Sigma}$. $\check{\Sigma}$ и $G . q$ являются регулярно вложенными подмногообразиями в $Y$ и тем самым они наследуют из $(Y, \eta)$ римановы метрики $\eta_{\Sigma}$ и $\eta_{G . q}$. Пусть $\Delta_{\Sigma}$ и $\Delta_{G . q}$ - операторы Лапласа-Бельтрами, определенные на соответствующих римановых многообразиях $\left(\check{\Sigma}, \eta_{\check{\Sigma}}\right)$ и $\left(G . q, \eta_{G . q}\right)$. Введем гладкую функцию плотности $\delta: \check{\Sigma} \rightarrow \mathbb{R}_{>0}:$

$$
\delta(q):=\text { объем риманова многообразия }\left(G . q, \eta_{G . q}\right) .
$$

Разумеется, объем понимается по отношению к мере, связанной с $\eta_{G . q}$. (По той же самой формуле функцию $\delta$ можно определить также как $W$-инвариантную функцию на $\Sigma$.) С помощью формулы (2.7) определим функцию $\mathcal{J}: \check{\Sigma} \rightarrow \operatorname{End}\left(\mathcal{K}^{\perp}\right)$ :

$$
\mathcal{J}:=\left.\mathcal{I}\right|_{\check{\Sigma}}
$$


и заметим, что из-за $G$-симметрии оператор инерции $\mathcal{J}(q)$ несет ту же информацию, что и метрика $\eta_{G . q}$. Пусть $\left\{T_{\alpha}\right\}$ и $\left\{T^{\beta}\right\}$ - некоторые фиксированные дуальные базисы в $\mathcal{K}^{\perp}$ по отношению к скалярному произведению $\mathcal{B}$,

$$
\mathcal{B}\left(T_{\alpha}, T^{\beta}\right)=\delta_{\alpha}^{\beta}
$$

В действительности (см. также замечание 3 ниже)

$$
\delta(q)=C\left|\operatorname{det} b_{\alpha, \beta}(q)\right|^{1 / 2} \quad \text { при } \quad b_{\alpha, \beta}(q)=\mathcal{B}\left(\mathcal{J}(q) T_{\alpha}, T_{\beta}\right)
$$

с не зависящей от $q$ константой $C>0$, значение которой известно, но оно для нас не важно.

ПреДЛОЖЕНИЕ. На $\operatorname{Fun}\left(\check{\Sigma}, V^{K}\right)$ (4.6) эффективный оператор Лапласа-Бельтрами (4.9) можно выразить в виде

$$
\Delta_{\text {eff }}=\delta^{-1 / 2} \circ \Delta_{\check{\Sigma}} \circ \delta^{1 / 2}-\delta^{-1 / 2} \Delta_{\check{\Sigma}}\left(\delta^{1 / 2}\right)+b^{\alpha, \beta} \rho^{\prime}\left(T_{\alpha}\right) \rho^{\prime}\left(T_{\beta}\right) .
$$

Более явным образом, это означает, что для любых $q \in \check{\Sigma} u f \in \operatorname{Fun}\left(\check{\Sigma}, V^{K}\right)$ имеем

$$
\begin{gathered}
\left(\Delta_{\mathrm{eff}} f\right)(q)=\delta^{-1 / 2}(q)\left(\Delta_{\check{\Sigma}}\left(\delta^{1 / 2} f\right)\right)(q)-\delta^{-1 / 2}(q)\left(\Delta_{\check{\Sigma}} \delta^{1 / 2}\right)(q) f(q)+ \\
+b^{\alpha, \beta}(q) \rho^{\prime}\left(T_{\alpha}\right) \rho^{\prime}\left(T_{\beta}\right) f(q),
\end{gathered}
$$

где линейный оператор на $V^{K}$ в последнем слагаемом содержит матрииу $b^{\alpha, \beta}(q)=$ $\mathcal{B}\left(\mathcal{J}^{-1}(q) T^{\alpha}, T^{\beta}\right)$, обратную $\kappa$ матрице $b_{\alpha, \beta}(q)(4.13)$.

ДоказАтельство. Возьмем произвольную функцию $\mathcal{F} \in C^{\infty}(Y, V)$ и рассмотрим ее ограничения

$$
f:=\left.\mathcal{F}\right|_{\check{\Sigma}}, \quad \mathcal{F}_{q}:=\left.\mathcal{F}\right|_{G . q} \quad \forall q \in \check{\Sigma} .
$$

Тогда $\left(\Delta_{Y} \mathcal{F}\right)(q)$ можно найти с помощью следующей хорошо известной формулы (4.17).

ЛЕмма 1. В приведенных выше обозначениях

$$
\left(\Delta_{Y} \mathcal{F}\right)(q)=\left(\Delta_{\text {rad }} f\right)(q)+\left(\Delta_{G . q} \mathcal{F}_{q}\right)(q)
$$

причем радиальная часть оператора $\Delta_{\check{Y}}$ есть

$$
\Delta_{\mathrm{rad}}=\delta^{-1 / 2} \circ \Delta_{\check{\Sigma}} \circ \delta^{1 / 2}-\delta^{-1 / 2} \Delta_{\check{\Sigma}}\left(\delta^{1 / 2}\right)
$$

Утверждение леммы 1 вполне стандартно (см., например, [24]). Его можно проверить и непосредственно, записывая оператор Лапласа-Бельтрами в таких локальных координатах $\left\{y^{a}\right\}=\left\{q^{i}\right\} \cup\left\{z^{\alpha}\right\}$ вблизи $q \in \check{\Sigma}$, которые составлены из некоторых координат $q^{i}$ на $\check{\Sigma}$ и координат $z^{\alpha}$ в окрестности начала координат в косет-пространстве $G / K$ в соответствии с $G$-эквивариантным диффеоморфизмом

$$
\check{\Sigma} \times G / K \simeq \check{Y}
$$


определенным как $\check{\Sigma} \times G / K \ni(q, g K) \mapsto \phi_{g}(q) \in \check{Y}$. Координаты можно выбрать, используя локальный диффеоморфизм

$$
\mathcal{K}^{\perp} \ni z=z^{\alpha} T_{\alpha} \mapsto e^{z} K \in G / K .
$$

Метрика на $Y$ тогда представляется как $g_{a, b}(q, z):=\eta_{(q, z)}\left(\partial_{y^{a}}, \partial_{y^{b}}\right)$, причем

$$
g_{i, j}(q, z)=g_{i, j}(q, 0), \quad g_{i, \alpha}(q, z)=0, \quad g_{\alpha, \beta}(q, 0)=b_{\alpha, \beta}(q)
$$

где $b_{\alpha, \beta}(q)$ определено в (4.13). В силу этой блочно-диагональной структуры локальное выражение

$$
\Delta_{Y} \leftrightarrow \frac{1}{\sqrt{\mathbf{g}}} \partial_{y^{a}} \circ \sqrt{\mathbf{g}} g^{a b} \circ \partial_{y^{b}}, \quad \mathbf{g}:=\left|\operatorname{det} g_{a, b}\right|,
$$

разбивается на сумму двух слагаемых, которые приводят к двум слагаемым в (4.17). Поскольку $f \in \operatorname{Fun}\left(\check{\Sigma}, V^{K}\right)$ отвечает $\mathcal{F} \in C_{c}^{\infty}(Y, V)^{G}$, для доказательства предложения теперь достаточно вычислить $\Delta_{G . q} \mathcal{F}_{q}$ для эквивариантных функций $\mathcal{F}_{q} \in$ $C^{\infty}(G . q, V)^{G}$. Результат последнего вычисления можно, по-видимому, также найти в литературе, но можно и получить его непосредственно, используя введенные выше экспоненциальные координаты на $G . q \simeq G / K$. Таким образом, имеем

$$
\left(\Delta_{G . q} \mathcal{F}_{q}\right)(q)=b^{\alpha, \beta}(q) \rho^{\prime}\left(T_{\alpha}\right) \rho^{\prime}\left(T_{\beta}\right) f(q), \quad \mathcal{F}_{q} \in C^{\infty}(G . q, V)^{G},
$$

что и завершает доказательство предложения.

ЗАмечание 3 . Косет-пространство $G / K$ несет $G$-инвариантную меру Хаара, которая единственна с точностью до мультипликативной постоянной. Мера Хаара ассоциированна с $G$-инвариантной дифференциальной формой максимальной степени на $G / K$. Эта дифференциальная форма единственным образом определяется своим значением в начале координат в $K \in G / K$. После отождествления $G / K \simeq G . q$ начало координат попадает в $q$, и значение $G$-инвариантной формы объема, определяемой метрикой $\eta_{G . q}$, дает в начале координат

$$
\left|\operatorname{det} b_{\alpha, \beta}(q)\right|^{1 / 2}\left(d z^{1} \wedge d z^{2} \wedge \cdots \wedge d z^{m}\right)_{q}, \quad m:=\operatorname{dim}(G / K) .
$$

Формула (4.13) легко следует из этого замечания.

4.2. Редуцированная квантовая система. Можно показать, что эффективный оператор Лапласа-Бельтрами $\Delta_{\text {eff }}(4.14)$ существенно самосопряжен на области $\operatorname{Fun}\left(\check{\Sigma}, V^{K}\right) \subset \overline{\operatorname{Fun}}\left(\check{\Sigma}, V^{K}\right) \simeq L^{2}\left(Y, V, d \mu_{Y}\right)^{G}$. Чтобы связать редуцированное гильбертово пространство с римановой метрикой на гладкой части редуцированного конфигурационного многообразия $\left(\check{Y}_{\text {red }}, \eta_{\text {red }}\right) \simeq\left(\check{\Sigma}, \eta_{\check{\Sigma}}\right)$, требуется следующая лемма.

Лемма 2. Дополнение плотного открытого подмногообразия $\check{Y} \subset Y$ типа главной орбиты имеет меру нуль относительно $d \mu_{Y}$. 
ДокАзАТЕльСтво. Известно [20], что неглавные орбиты данного типа заполняют регулярные подмногообразия меньшей размерности в $Y$, причем существует не более чем счетное число различных типов орбит. Из того, что мера $d \mu_{Y}$ гладкая, а $Y$ удовлетворяет второй аксиоме счетности, следует (см., например, [25], с. 529), что $Y \backslash \check{Y}$ имеет меру нуль.

Далее нам потребуется следующая формула интегрирования:

$$
\begin{gathered}
\int_{Y}\left(\mathcal{F}_{1}, \mathcal{F}_{2}\right)_{V} d \mu_{Y}=\int_{\check{Y}}\left(\mathcal{F}_{1}, \mathcal{F}_{2}\right)_{V} d \mu_{\check{Y}}=\int_{\check{\Sigma}}\left(f_{1}, f_{2}\right)_{V} \delta d \mu_{\check{\Sigma}}, \\
\mathcal{F}_{i} \in C_{c}^{\infty}(Y, V)^{G}, \quad f_{i}=\left.\mathcal{F}_{i}\right|_{\check{\Sigma}} .
\end{gathered}
$$

Первое равенство следует из леммы 2. Второе выполняется потому, что мера на $\check{Y} \simeq \check{\Sigma} \times G / K$, как можно показать стандартными средствами, принимает вид меры для произведения

$$
d \mu_{\check{Y}}=\left(\delta d \mu_{\check{\Sigma}}\right) \times d \mu_{G / K},
$$

где $d \mu_{G / K}$ - вероятностная мера Хаара на $G / K, d \mu_{\check{\Sigma}}$ - мера на $\check{\Sigma}$, определяемая римановой метрикой $\eta_{\Sigma}$, плотность $\delta$ определена в $(4.10)$, а $\left(\mathcal{F}_{1}, \mathcal{F}_{2}\right)_{V}$ есть $G$-инвариант. Формула интегрирования и то обстоятельство, что $\operatorname{Fun}\left(\check{\Sigma}, V^{K}\right)$ содержит $C_{c}^{\infty}\left(\check{\Sigma}, V^{K}\right)$ (причем $\left.C_{c}^{\infty}(\check{Y}, V)^{G} \subset C_{c}^{\infty}(Y, V)^{G}\right)$, приводят к соотношению

$$
\overline{\operatorname{Fun}}\left(\check{\Sigma}, V^{K}\right) \simeq L^{2}\left(\check{\Sigma}, V^{K}, \delta d \mu_{\check{\Sigma}}\right)
$$

Удаляя преобразованиями множитель $\delta$ из “индуцированной меры” $\delta d \mu_{\check{\Sigma}}$, получаем окончательный результат.

Теорема 2. В приведенных выше обозначениях редукиия квантовой системы, определяемой замыканием оператора $-\Delta_{Y} / 2$ на $C_{c}^{\infty}(Y, V) \subset L^{2}\left(Y, V, d \mu_{Y}\right)$, приводит $к$ редуиированному оператору Гамильтона $-\Delta_{\mathrm{red}} / 2$ вида

$$
\Delta_{\text {red }}=\delta^{1 / 2} \circ \Delta_{\text {eff }} \circ \delta^{-1 / 2}=\Delta_{\check{\Sigma}}-\delta^{-1 / 2}\left(\Delta_{\check{\Sigma}} \delta^{1 / 2}\right)+b^{\alpha, \beta} \rho^{\prime}\left(T_{\alpha}\right) \rho^{\prime}\left(T_{\beta}\right) .
$$

Этот оператор является существенно самосопряженным на плотной области $\delta^{1 / 2} \operatorname{Fun}\left(\check{\Sigma}, V^{K}\right)$ в редуцированном гильбертовом пространстве, отождествляемом $\operatorname{\kappa a\kappa } L^{2}\left(\check{\Sigma}, V^{K}, d \mu_{\check{\Sigma}}\right)$.

ДокАЗАтЕЛЬство. Оператор умножения $U: f \mapsto \delta^{1 / 2} f$ является изометрией из $L^{2}\left(\check{\Sigma}, V^{K}, \delta d \mu_{\check{\Sigma}}\right)$ в $L^{2}\left(\check{\Sigma}, V^{K}, d \mu_{\check{\Sigma}}\right)$. Из предложения и леммы 2 следует, что $\Delta_{\text {red }}=$ $U \circ \Delta_{\text {eff }} \circ U^{-1}$ - симметричный оператор на плотной области $U\left(\operatorname{Fun}\left(\check{\Sigma}, V^{K}\right)\right)=$ $\delta^{1 / 2} \operatorname{Fun}\left(\check{\Sigma}, V^{K}\right) \subset L^{2}\left(\check{\Sigma}, V^{K}, d \mu_{\check{\Sigma}}\right) . \quad$ Существенная самосопряженность оператора $\Delta_{\text {red }}$ имеет место из-за существенной самосопряженности оператора $\Delta_{Y}$ на $C_{c}^{\infty}(Y, V)$. Дальнейшие подробности по этому вопросу приведены в [26].

Сравним результат квантовой гамильтоновой редукции в теореме 2 с классической редуцированной системой из теоремы 1. Прежде всего, классическая кинетическая энергия, очевидно, соответствует $-\Delta_{\check{\Sigma}} / 2$. Формально, второе слагаемое в классическом гамильтониане $\mathcal{H}_{\text {red }}(3.10)$ соответствует третьему слагаемому $-\Delta_{\text {red }} / 2$ (4.28). Это слагаемое можно интерпретировать как потенциальную 
энергию, если $\operatorname{dim}\left(V^{K}\right)=1$, в противном же случае оно представляет собой зависящую от спина потенциальную энергию. Представленный вторым слагаемым в (4.28) дополнительный “множитель" для меры появляется, вообще говоря, на квантовом уровне, но никак не проявляет себя в $\mathcal{H}_{\text {red. }}$ Это слагаемое дает постоянный или нетривиальный вклад в потенциальную энергию в зависимости от конкретных примеров.

В квантовой гамильтоновой редукции мы исходили из полного конфигурационного пространства $Y$, тогда как в классическом случае мы ограничились на пространстве $\check{Y}$ с самого начала. В некотором смысле результат квантовой гамильтоновой редукции можно тем не менее рассматривать как квантование редуцированной классической системы из теоремы 1 , поскольку $Y \backslash \check{Y}$ имеет меру нуль. Однако это соответствие требует дальнейшего исследования (см. также [27]). Также должна быть изучена структура полного (сингулярного) редуцированного фазового пространства $P_{\text {red }}$, происходящего из $T^{*} Y$, поскольку ясно, что редуцированные

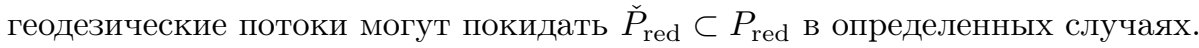

Подчеркнем, что на абстрактном уровне в силу естественных отождествлений (4.7) и (4.27) редуцированное гильбертово пространство просто порождается $G$-синглетами $L^{2}\left(Y, V, d \mu_{Y}\right)^{G}$. В некоторых случаях (например, если $Y$ - компактная группа Ли) $\Delta_{Y}$ имеет чисто точечный спектр, который можно найти с помощью известных результатов гармонического анализа (таких как теорема Петера-Вейля). В таких случаях нахождение спектра редуцированного оператора Гамильтона становится задачей на "branching-правила", поскольку требуется найти указанные выше $G$-синглеты среди собственных подпространств оператора $\Delta_{Y}$.

\section{5. ПРИМЕРЫ, СВЯЗАННЫЕ С МОДЕЛЯМИ ТИПА МОДЕЛИ САЗЕРЛЕНДА СО СПИНОМ}

Вспомним (см. [17], [18]) о классе важных гиперполярных действий на компактных группах Ли. Применяя к ним классическую и квантовую гамильтонову редукцию, как описано в данной работе, можно получить большое семейство интегрируемых моделей типа модели Сазерленда со спином. Подробности, относящиеся к этим моделям, будут представлены в другой публикации.

Пусть $Y$ - компактная связная полупростая группа Ли с римановой метрикой, индуцированной формой, кратной форме Киллинга. Возьмем в качестве группы редукции $G$ любую симметричную подгруппу в $Y \times Y$. Другими словами, $G$ - любая подгруппа, которая остается поточечно неподвижной под действием инволютивного автоморфизма $\sigma$ группы $Y \times Y$ и содержит связную компоненту полной подгруппы, оставляемой неподвижной автоморфизмом $\sigma$. Рассмотрим следующее действие $G$ на $Y$ :

$$
\phi_{(a, b)}(y):=a y b^{-1} \quad \forall y \in Y, \quad(a, b) \in G \subset Y \times Y .
$$

Известно, что это действие гиперполярно (см. [17], [18] и приведенную там библиографию). Сечения даются определенными торами $A \subset Y$. Действительно $A$ есть экспонента от абелевой подалгебры $\mathcal{A}$ правильной размерности, содержащейся в подпространстве $\left(T_{e}(G . e)\right)^{\perp}$ пространства $T_{e} Y$. Причины, обусловливающие появление 
моделей типа модели Сазерленда в связи с этими действиями, состоят в экспоненциальной параметризации $\Sigma=A$ вместе с разложением алгебры Ли группы $Y$ на совместные собственные подпространства подалгебры $\mathcal{A}$. Это можно проиллюстрировать на частных примерах, к которым мы и обратимся.

Во-первых, рассмотрим $\sigma\left(y_{1}, y_{2}\right)=\left(y_{2}, y_{1}\right)$. Тогда $G=\{(a, a) \mid a \in Y\} \simeq Y$ и действие (5.1) есть просто присоединенное действие группы $Y$ на себе, для которого сечения суть максимальные торы в $Y$. Ассоциированные модели Сазерленда со спином (а в некоторых исключительных случаях - и без спина) изучались в [2], [7].

Во-вторых, возьмем любой нетривиальный автоморфизм $\theta$ группы $Y$ и положим $\sigma\left(y_{1}, y_{2}\right):=\left(\theta\left(y_{2}\right), \theta^{-1}\left(y_{1}\right)\right)$. Тогда

$$
G=\{(\theta(a), a) \mid a \in Y\} \simeq Y,
$$

и (5.1) дает действие группы $Y$ на себе посредством $\theta$-твистованных сопряжений $\phi_{(\theta(a), a)}(y)=\theta(a) y a^{-1}$. Интересны случаи, когда $\theta$ соответствует симметрии диаграммы Дынкина группы $Y$. Некоторые из получающихся обобщенных моделей Сазерленда со спином описаны в [11], [28].

В-третьих, предположим, что $\theta_{1}$ и $\theta_{2}$ - два инволютивных автоморфизма группы $Y$, и пусть $K_{1}$ и $K_{2}$ - соответствующие симметричные подгруппы в $Y$, т.е. $\left(Y, K_{j}\right)$ - симметричные пары при $j=1,2$. Полагая $\sigma\left(y_{1}, y_{2}\right):=\left(\theta_{1}\left(y_{1}\right), \theta_{2}\left(y_{2}\right)\right)$, получаем

$$
G=K_{1} \times K_{2} \subset Y \times Y,
$$

и (5.1) принимает вид так называемого действия Херманна на $Y$. Помимо этого действия, индуцированное действие $K_{1}$ на $Y / K_{2}$ также является гиперполярным. Получающиеся модели типа модели Сазерленда со спином систематически пока не изучались, за исключением случая [12] изотропного действия $K_{1}$ на симметрическом пространстве $Y / K_{1}$, возникающего при $K_{1}=K_{2}$. Поскольку они в действительности содержат интересные бесспиновые случаи, мы вернемся к моделям этого типа в другой публикации. Перспективным может оказаться исследование редуцированных систем, индуцированных другими полярными действиями из работ [17], [18].

Из-за компактности $Y$ в отмеченных выше случаях для соответствующих моделей Сазерленда потенциалы содержат тригонометрические функции. Гиперболические аналоги этих моделей можно вывести [13] исходя из некомпактных полупростых групп Ли $Y$. Это требует некоторого обобщения теории полярных действий, распространяемого на подходящие действия на псевдоримановых многообразиях. Рациональные вырождения тригонометрических моделей можно получить гамильтоновой редукцией в тех случаях, когда $G$-действие имеет неподвижную точку $p$, используя тот факт, что тогда представление группы $G$ на $T_{p} Y$ наследует свойство полярности исходного действия. Важная открытая проблема состоит в выяснении того, можно ли так расширить формализм гамильтоновой редукции относительно полярных действий, чтобы включить в теорию и эллиптические модели типа модели Калоджеро-Сазерленда.

Благодарности. Л. Фехер выражает благодарность З. Хохгернеру и Л. Верхочки за полезные обсуждения. Б. Пустай благодарит Ж. Харна за гостеприимство 
в Монреале. Работа Л. Фехера частично поддержана Hungarian Scientific Research Fund (OTKA грант T049495) и EU network "ENIGMA" (контракт MRTN-CT-2004$5652)$.

\section{Список литературы}

[1] D. Kazhdan, B. Kostant, S. Sternberg, Comm. Pure Appl. Math., 31:4 (1978), 481-507.

[2] P. I. Etingof, I. B. Frenkel, A. A. Kirillov Jr., Duke Math. J., 80:1 (1995), 59-90; arXiv: hep-th/9407047.

[3] M. A. Olshanetsky, A. M. Perelomov, Phys. Rep., 71:5 (1981), 313-400.

[4] M. A. Olshanetsky, A. M. Perelomov, Phys. Rep., 94:6 (1983), 313-404.

[5] J. Gibbons, T. Hermsen, Physica D, 11:3 (1984), 337-348.

[6] N. Nekrasov, "Infinite-dimensional algebras many-body systems and gauge theories", Moscow Seminar in Mathematical Physics, AMS Transl. Ser. 2, 191, eds. A. Yu. Morozov, M. A. Olshanetsky, Amer. Math. Soc., Providence, RI, 1999, 263-299.

[7] N. Reshetikhin, Lett. Math. Phys., 63:1 (2003), 55-71; arXiv: math.QA/0202245.

[8] D. Alekseevsky, A. Kriegl, M. Losik, P. W. Michor, Publ. Math. Debrecen, 62:3-4 (2003), 247-276; arXiv: math.DG/0102159.

[9] A. Oblomkov, Adv. Math., 186:1 (2004), 153-180; arXiv: math.RT/0202076.

[10] S. Hochgerner, Singular cotangent bundle reduction and spin Calogero-Moser systems, arXiv: math.SG/0411068.

[11] L. Fehér, B. G. Pusztai, Nucl. Phys. B, 734:3 (2006), 304-325; arXiv: math-ph/0507062.

[12] L. Fehér, B. G. Pusztai, Nucl. Phys. B, 751:3 (2006), 436-458; arXiv: math-ph/0604073.

[13] L. Fehér, B. G. Pusztai, Lett. Math. Phys., 79:3 (2007), 263-277; arXiv: math-ph/0609085.

[14] R. Palais, C.-L. Terng, Trans. Amer. Math. Soc., 300:2 (1987), 771-789.

[15] L. Conlon, J. Differential Geom., 5 (1971), 135-147.

[16] J. Szenthe, Period. Math. Hungar., 15:4 (1984), 281-299.

[17] E. Heintze, R. Palais, C.-L. Terng, G. Thorbergsson, "Hyperpolar actions on symmetric spaces", Geometry, Topology and Physics for Raoul Bott, Conf. Proc. Lecture Notes Geom. Topology, IV, ed. S.-T. Yau, International Press, Cambridge, MA, 1995, 214-245.

[18] A. Kollross, Polar actions on symmetric spaces, arXiv: math.DG/0506312.

[19] S. Hochgerner, Spinning particles in a Yang-Mills field, arXiv: math.SG/0602062.

[20] А.Л. Онищик, Р. В. Гамкрелидзе (ред.), Группы Ли и алгебры Ли 1, Итоги науки и техники. Соврем. проблемы матем. Фундам. напр., 20, ВИНИТИ, 1988.

[21] J.-P. Ortega, T. S. Ratiu, Momentum Maps and Hamiltonian Reduction, Progr. Math., 222, Birkhäuser, Boston, MA, 2004.

[22] S. Tanimura, T. Iwai, J. Math. Phys., 41:4 (2000), 1814-1842; arXiv: math-ph/9907005.

[23] N. P. Landsman, Mathematical Topics Between Classical and Quantum Mechanics, Springer Monogr. Math., Springer, New York, 1998.

[24] S. Helgason, Groups and Geometric Analysis, Pure Appl. Math., 113, Academic Press, Orlando, FL, 1984.

[25] A. W. Knapp, Lie Groups Beyond an Introduction, Progr. Math., 140, Birkhäuser, Boston, MA, 2002.

[26] L. Fehér, B. G. Pusztai, On the self-adjointness of certain reduced Laplace-Beltrami operators, arXiv: 0707.2708 (to appear in Rep. Math. Phys.).

[27] G. Kunstatter, Class. Quant. Grav., 9:6 (1992), 1469-1485.

[28] L. Fehér, B. G. Pusztai, Twisted spin Sutherland models from quantum Hamiltonian reduction, arXiv: 0711.4015, submitted to J. Phys. A. 